\title{
AVERAGES ALONG UNIFORMLY DISTRIBUTED DIRECTIONS ON A CURVE
}

\author{
JOSE BARRIONUEVO
}

(Communicated by J. Marshall Ash)

\begin{abstract}
We obtain a sharp $L^{2}$ estimate for the maximal operator associated with uniformly distributed directions on a curve of finite type in $\mathbf{R}^{n}$.
\end{abstract}

\section{INTRODUCTION}

Let $\gamma:[0,1] \rightarrow S^{n-1}$ be a smooth curve crossing each hyperplane of $\mathbf{R}^{n}$ a finite number of times. If $\mathscr{B}_{N}$ denotes the family of all cylinders in $\mathbf{R}^{n}$ having eccentricity $N$ and direction in $\gamma$, it is proved in [C] that the maximal operator

$$
M_{N} f(x)=\sup _{x \in R \in \mathscr{B}_{N}} \frac{1}{|R|} \int_{R}|f(y)| d y
$$

satisfies the estimate

$$
\left\|M_{N} f\right\|_{L^{2}} \leq C_{\gamma}(\log N)^{2}\|f\|_{L^{2}}
$$

where $C_{\gamma}$ is independent of $N$.

The purpose of this note is to show that by imposing an additional condition on $\gamma$ one can prove a stronger result.

Let $\gamma$ be a smooth curve satisfying

$$
\text { For all } t \in[0,1] \text {, the set }\left\{\gamma^{(j)}(t)\right\}_{0 \leq j<\infty} \text { spans } \mathbf{R}^{n} \text {. }
$$

For a positive integer $m$ let $\mathscr{B}_{m}$ denote the family of all cylinders in $\mathbf{R}^{n}$ pointing in the direction of $\gamma\left(j / 2^{m}\right)$ for some $0 \leq j \leq 2^{m}$. let $\mathscr{M}_{m} f(x)=$ $\sup _{x \in R \in \mathscr{B}_{m}}(1 /|R|) \int_{R}|f(y)| d y$. Then we will prove the following:

Theorem. If $\gamma$ satisfies $(*)$ then

$$
\left\|\mathscr{M}_{m} f\right\|_{L^{2}} \leq C_{\gamma} m\|f\|_{L^{2}}
$$

where $C_{\gamma}$ is independent of $m$.

If $n=2$ or if $\gamma$ is contained in a 2-dimensional subspace, (2) is known to be true (see [S] or [B]). Also, since $\mathscr{M}_{m}$ dominates $M_{2^{m}},(2)$ implies an improved version of (1).

In what follows all the constants are independent of $m$.

Received by the editors March 4, 1991 and, in revised form, March 9, 1992.

1991 Mathematics Subject Classification. Primary 42B10. 


\section{AUXILIARY LEMMAS}

We will now prove some consequences of $(*)$ that will be used to prove the theorem.

A simple compactness argument shows that if $\gamma$ satisfies $(*)$, then there exist an integer $L$ and $c>0$ such that for all $\xi \in S^{n-1}$ and $t \in[0,1]$

$$
\sum_{i=0}^{L}\left|\xi \cdot \gamma^{(i)}(t)\right| \geq c
$$

For $j=0,1,2$ let $\mathscr{U}_{j}=\left\{(\xi, t) \in S^{n-1} \times[0,1]:\left|\xi \cdot \gamma^{(l)}(t)\right| \leq c 2^{-(l+1)}\right.$ for $l \leq j\}$. Then we have

Lemma 1. There exist $\delta_{j}>0$ and $c_{j}>0$ such that for all $(\xi, t) \in \mathscr{U}_{j}$

$$
|s-t|<\delta_{j} \Rightarrow\left|\xi \cdot\left(\gamma^{(j)}(s)-\gamma^{(j)}(t)\right)\right| \geq c_{j}|s-t|^{L-j} .
$$

Proof. If the lemma is false, we can find sequences $\varepsilon_{k} \rightarrow 0, \delta_{k} \rightarrow 0,\left(\xi_{k}, t_{k}\right) \in$ $S^{n-1} \times[0,1]$, and $s_{k}$ such that $\left|s_{k}-t_{k}\right|<\delta_{k}$ and

$$
\left|\xi_{k} \cdot\left(\gamma^{(j)}\left(s_{k}\right)-\gamma^{(j)}\left(t_{k}\right)\right)\right|<\varepsilon_{k}\left|s_{k}-t_{k}\right|^{L-j} .
$$

Since $\mathscr{U}_{j}$ is compact, by passing to a subsequence, we can assume that $\left(\xi_{k}, t_{k}\right)$ converges to $(\xi, i) \in \mathscr{U}_{j}$. By Taylor's theorem (5) implies that $\xi \cdot \gamma^{(l)}(t)=0$ for $l=j+1, \ldots, L$. This contradicts $(3)$.

Lemma 1 implies that there exist integers $N_{j}\left(\sim \delta_{j}^{-1}\right)$ such that for all $\xi$ in $S^{n-1}$ the function $\xi \cdot \gamma^{(j)}(t)$ has at most $N_{j}$ zeros on $\left\{t \in[0,1]:\left|\xi \cdot \gamma^{(l)}(t)\right|<\right.$ $c 2^{-(l+1)}$ for $\left.0 \leq l \leq j-1\right\}$.

For $\xi \in S^{n-1}$ let $v_{\xi}(t)=\xi \cdot \gamma(t), \mathscr{V}_{\xi}^{1}=\left\{t \in[0,1]:\left|v_{\xi}(t)\right|>c / 2\right\}$, and $\mathscr{V}_{\xi}^{2}=\left\{t \in[0,1]:\left|v_{\xi}(t)\right|<c / 2\right.$ and $\left.\left|v_{\xi}^{\prime}(t)\right|>c / 4\right\}$. Since $\mathscr{V}_{\xi}^{1}$ and $\mathscr{V}_{\xi}^{2}$ are open (in $[0,1]$ ) and disjoint, we can write each $\mathscr{V}_{\xi}^{j}$ as a countable union of disjoint intervals. Since between each two intervals of $\mathscr{V}_{\xi}^{1}$ there exists a $t$ for which either $v_{\xi}(t)=0$ or $v_{\xi}^{\prime}(t)=0$, Lemma 1 implies that $\mathscr{V}_{\xi}^{1}$ is the union of at most $N_{0}+N_{1}$ (independent of $\xi$ ) intervals. A similar argument applied to $\mathscr{V}_{\xi}^{2}$ in the complement of $\mathscr{V}_{\xi}^{1}$ together with the fact that, on the complement of $\mathscr{V}_{\xi}^{1} \cup \mathscr{V}_{\xi}^{2}, v_{\xi}^{\prime \prime}(t)$ has at most $N_{2}$ zeros shows that the complement of $\mathscr{V}_{\xi}^{1} \cup \mathscr{V}_{\xi}^{2}$ can be written as a union of no more than $2\left(N_{0}+N_{1}+N_{2}\right)$ closed intervals where, on each of these, $v_{\xi}^{\prime}(t)$ is monotonic. Let $I=[a, b]$ be one such interval, and let $t_{0} \in[a, b]$ be such that $\left|v_{\xi}^{\prime}\left(t_{0}\right)\right|=\min _{I}\left|v_{\xi}^{\prime}(t)\right|$. Then we have

$$
\begin{aligned}
v_{\xi}^{\prime}(t) & =\sum_{j=0}^{L-1} \frac{v_{\xi}^{(j+1)}\left(t_{0}\right)}{j !}\left(t-t_{0}\right)^{j}+R_{t_{0}}(t) \\
& =p_{\xi}^{\prime}(t)+R_{t_{0}}(t) \quad \text { where }\left|R_{t_{0}}(t)\right| \leq C\left|t-t_{0}\right|^{L} .
\end{aligned}
$$

Thus if $\delta_{\gamma}=1 / 2 \min \left\{\min _{j} \delta_{j}, c_{1} C^{-1}\right\}$ we have for $\left|t-t_{0}\right|<\delta_{\gamma}$ and $t \neq t_{0}$

$$
\left|\frac{p_{\xi}^{\prime}(t)}{v_{\xi}^{\prime}(t)}-1\right| \leq \frac{C\left|t-t_{0}\right|^{L}}{\left|v_{\xi}^{\prime}(t)\right|} \leq \frac{1}{2},
$$


which implies

$$
\left|p_{\xi}^{\prime}(t)\right| \leq\left|v_{\xi}^{\prime}(t)\right| \leq 2\left|p_{\xi}^{\prime}(t)\right| .
$$

If we let $p_{\xi}^{\prime}(t)-v_{\xi}^{\prime}\left(t_{0}\right)=q_{\xi}(t)$, we have by Lemma 1 that there exist $c_{\xi}>0$ such that $\left|q_{\xi}(t)\right| \approx c_{\xi}\left|t-t_{0}\right|^{k}$ for $\left|t-t_{0}\right|<\delta_{\gamma}$ and for some $k$ with $1 \leq k \leq L-1$. If $\left|t-t_{0}\right|>\delta_{\gamma}$ and $t \in I$, Lemma 1 implies that $\left|v_{\xi}^{\prime}(t)\right| \geq c_{1} \delta_{\gamma}^{L-1}$. Since $v_{\xi}(t)$ has at most two zeros on $I$, we can divide $\left\{t \in I:\left|t-t_{0}\right|<\delta_{\gamma}\right\}$ in no more than four intervals where $v_{\xi}(t)$ is monotonic and of constant sign satisfying estimates like the above. Thus, if we let $N_{\gamma}=10\left(N_{0}+N_{1}+N_{2}\right)$ and $c_{\gamma}=\min \left\{c / 4, c_{1} \delta_{\gamma}^{L-1}\right\}$, we obtain

Lemma 2. There exist an integer $N_{\gamma}$ and $c_{\gamma}>0$ such that for all $\xi$ in $S^{n-1}$ we have

$$
[0,1]=U_{\xi}^{1} \cup \cdots \cup U_{\xi}^{N_{\xi}} \cup V_{\xi}^{1} \cup \cdots \cup V_{\xi}^{M_{\xi}} \cup W_{\xi}^{1} \cup \cdots \cup W_{\xi}^{K_{\xi}}
$$

where $N_{\xi}+M_{\xi}+K_{\xi} \leq N_{\gamma}$ and where the $U_{\xi}^{i}$ 's, $V_{\xi}^{i}$ 's, and $W_{\xi}^{i}$ 's are closed intervals with disjoint interiors for which

(i) $\left|v_{\xi}(t)\right| \geq c_{\gamma}$ on $\bigcup_{i} U_{\xi}^{i}$,

(ii) $\left|v_{\xi}^{\prime}(t)\right| \geq c_{\gamma}$ on $\bigcup_{i} V_{\xi}^{i}$, and

(iii) for each $i \leq K_{\xi}$ there exist $c_{\xi}^{i}>0, t_{0} \in W_{\xi}^{i}$, and $k=k_{\xi, i, t_{0}}$ with $1 \leq k \leq L$ such that

$$
\left|v_{\xi}(t)\right| \approx\left|v_{\xi}\left(t_{0}\right)\right|+c_{\xi}^{i}\left|t-t_{0}\right|^{k} \quad \text { and } \quad\left|v_{\xi}^{\prime}(t)\right| \approx c_{\xi}^{k} k\left|t-t_{0}\right|^{k-1} \text {. }
$$

Proof of Theorem. The proof is based in a square function argument following the ideas in [W, NSW].

Let $\varphi \in C_{0}^{\infty}(\mathbf{R})$ be nonnegative, with $\varphi \equiv 1$ on $\left[-\frac{1}{4}, \frac{1}{4}\right]$ and such that $\int_{-\infty}^{\infty} \varphi(t) d t=1$. For $h>0$ let $\varphi_{h}(t)=h^{-1} \varphi\left(h^{-1} t\right)$ and let $\omega_{j}=\gamma\left(j 2^{-m}\right)$.

For $0 \leq j \leq 2^{m}$ let

$$
T_{h, j}^{m} f(x)=\int_{-\infty}^{\infty} f\left(x-t \omega_{j}\right) \varphi_{h}(t) d t, \quad T^{m} f(x)=\sup _{h, j}\left|T_{h, j}^{m} f(x)\right| .
$$

Then a simple geometric argument shows that it suffices to prove that

$$
\left\|T^{m} f\right\|_{L^{2}} \leq C_{\gamma} m\|f\|_{L^{2}} \text { for } f \geq 0 .
$$

For $m=1,(6)$ follows from the boundedness of the one-dimensional HardyLittlewood maximal operator. Suppose (6) is true for $m=1$. Then for $f \geq 0$

$$
\begin{aligned}
T^{m} f(x) & \leq T^{m-1} f(x)+\sup _{h, j}\left|T_{h, 2 j}^{m} f(x)-T_{h, 2 j-1}^{m} f(x)\right| \\
& =T^{m} f(x)+\sup _{h, j} H_{h, j}^{m} f(x)
\end{aligned}
$$

and (6) will follow if we can show that

$$
\left\|\sup _{h, j} H_{h, j}^{m} f\right\|_{L^{2}} \leq C_{\gamma}\|f\|_{L^{2}}
$$

For $j=1, \ldots, 2^{m}$, let $\Gamma_{j}$ be the cone $\left\{\xi \in \mathbf{R}^{n}:\left|\xi \cdot \omega_{j}\right|>c_{1} 2^{-m L}|\xi|\right\}$ and let $K_{j}$ be the complement of $\Gamma_{j}$, and for $j=1, \ldots, 2^{m-1}$, let $f(x)=f_{j}(x)+r_{j}(x)$ where $\hat{r}_{j}(\xi)=\chi_{K_{2 j} \cup K_{2 j-1}}(\xi) \hat{f}(\xi)$. ` denotes the Fourier transform. 
An argument similar to the one in $[\mathrm{W}, \mathrm{p} .88]$ shows that

$$
\sup _{h, j} H_{h, j}^{m} f(x) \leq C\left(g_{1}(f)(x)+g_{2}(f)(x)\right)
$$

where

$$
\begin{aligned}
& g_{1}(f)(x)=\left(\sum_{j=1}^{2^{m-1}}\left(\sup _{h} T_{h, 2 j}^{m}\left|r_{j}(x)\right|+\sup _{h} T_{h, 2 j-1}^{m}\left|r_{j}(x)\right|\right)^{2}\right)^{1 / 2}, \\
& g_{2}(f)(x)=\left(\int_{0}^{\infty} \sum_{j=1}^{2^{m-1}}\left|T_{h, 2 j}^{m} f_{j}(x)-T_{h, 2 j-1}^{m} f_{j}(x)\right|^{2} \frac{d h}{h}\right)^{1 / 2} .
\end{aligned}
$$

Thus (8) and hence the theorem will be a consequence of the following two estimates:

$$
\begin{gathered}
\left\|g_{1}(f)\right\|_{L^{2}} \leq C_{\gamma}\|f\|_{L^{2}}, \\
\left\|g_{2}(f)\right\|_{L^{2}} \leq C_{\gamma}\|f\|_{L^{2}} .
\end{gathered}
$$

Proof of (9). By the boundedness of the one-dimensional Hardy-Littlewood maximal operator and Plancherel's theorem, one has

$$
\left\|g_{1}(f)\right\|_{L^{2}}^{2} \leq C \int_{\mathbf{R}^{n}} \sum_{j=1}^{2^{m-1}}\left|r_{j}(x)\right|^{2} d x=C \int_{\mathbf{R}^{n}} \sum_{j=1}^{2^{m-1}} \chi_{K_{2 j} \cup K_{2 j-1}}(\xi)|\hat{f}(\xi)|^{2} d \xi .
$$

Since the $K_{j}$ 's are conic, it is enough to prove that no $\xi \in S^{n-1}$ belongs to more than $C_{\gamma}$ of the $K_{j}$ 's. Given $\xi$ in $K_{j_{0}}$ we have $\left|v_{\xi}(t)\right| \leq c_{1} 2^{-m L}$. By (4), if $k>c_{1}^{1 / L}$, then $\xi$ does not belong to $K_{j_{0} \pm k}$. Thus $\xi$ does not belong to more than $N_{\gamma} c_{1}^{1 / L}$ of the $K_{j}$ 's.

Proof of (10). Plancherel's theorem implies that

$$
\begin{aligned}
\left\|g_{2}(f)\right\|_{L^{2}}^{2} & =\int_{\mathbf{R}^{n}} \sum_{j=1}^{2^{m-1}} \int_{0}^{\infty}\left|\hat{\varphi}\left(h \xi \cdot \omega_{2 j}\right)-\hat{\varphi}\left(h \xi \cdot \omega_{2 j-1}\right)\right|^{2}\left|\hat{f}_{j}(\xi)\right|^{2} \frac{d h}{h} d \xi \\
& =\int_{\mathbf{R}^{n}} m(\xi)|\hat{f}(\xi)|^{2} d \xi
\end{aligned}
$$

where

$$
m(\xi)=\sum_{j=1}^{2^{m-1}} \int_{0}^{\infty}\left|\hat{\varphi}\left(h \xi \cdot \omega_{2 j}\right)-\hat{\varphi}\left(h \xi \cdot \omega_{2 j-1}\right)\right|^{2} \chi_{\Gamma_{2 j} \cap \Gamma_{2 j-1}}(\xi) \frac{d h}{h},
$$

and we are left to prove that $m(\xi) \leq C_{\gamma}$. This is accomplished by dividing the curve $\gamma$ in pieces where one has control over the decay of $\hat{\varphi}\left(h \xi \cdot \omega_{j}\right)$ in $\xi$ and $j$ in estimating (13). The details are below.

By Lemma 2 we can, for each $\xi$, split the sum in (13) in no more than $N_{\gamma}$ sums of the form $\sum_{j 2-m \in U_{\xi}^{i}}, \sum_{j 2-m \in V_{\xi}^{i}}$, and $\sum_{j 2^{-m} \in W_{\xi}^{i}}$. Thus the theorem will follow if we can show that each of these sums is bounded with bound independent of $m$ and $\xi$. By homogeneity we only need to consider $\xi \in S^{n-1}$. 
Since $\left|\xi \cdot \omega_{j}\right| \geq c_{\gamma}$ for $j 2^{-m} \in U_{\xi}^{i}$, and since $\hat{\varphi}$ is a Schwartz function, we have that $\left|\hat{\varphi}\left(h \xi \cdot \omega_{j+1}\right)-\hat{\varphi}\left(h \xi \cdot \omega_{j}\right)\right|^{2} \leq C h^{2}\left|\omega_{j+1}-\omega_{j}\right|^{2}\left|\hat{\varphi}^{\prime}\left(h \xi \cdot u_{j}\right)\right|^{2}$ with $\left|\xi \cdot u_{j}\right| \geq c_{\gamma}$. This implies

$$
\sum_{j 2^{-m} \in U_{\xi}^{i}} \int_{0}^{\infty}\left|\hat{\varphi}\left(h \xi \cdot \omega_{j+1}\right)-\hat{\varphi}\left(h \xi \cdot \omega_{j}\right)\right|^{2} \frac{d h}{h} \leq C_{\gamma} .
$$

We now prove a similar estimate for $W_{\xi}^{i}$. There is no lack of generality in assuming that $W_{\xi}^{i}=[0, \varepsilon]$ and that $v_{\xi}(0)=0$. Lemma 2 implies that for $j 2^{-m} \in W_{\xi}^{i}$

$$
\begin{aligned}
\left|\xi \cdot\left(\omega_{2 j}-\omega_{2 j-1}\right)\right| & \leq C c_{\xi} j^{k-1} 2^{-m k}, \\
\left|\xi \cdot \omega_{j}\right| & \geq C c_{\xi} j^{k} 2^{-m k} .
\end{aligned}
$$

Since $\hat{\varphi}$ is smooth and rapidly decreasing, by (15) and (16) we obtain

$$
\begin{gathered}
\left|\hat{\varphi}\left(h \xi \cdot \omega_{2 j}\right)-\hat{\varphi}\left(h \xi \cdot \omega_{2 j-1}\right)\right|^{2} \leq C c_{\xi}^{2} j^{2(k-1)} 2^{-2 m k} h^{2}, \\
\left|\hat{\varphi}\left(h \xi \cdot \omega_{j}\right)\right|^{2} \leq C_{\alpha} c_{\xi}^{-2 \alpha} j^{-2 k \alpha} 2^{-2 m k \alpha} h^{-2 \alpha} .
\end{gathered}
$$

Spliting each integral in $\int_{0}^{a_{j}}+\int_{a_{j}}^{\infty}$ where the $a_{j}$ 's are to be determined later and using (17) and (18) on each integral respectively we obtain that $\sum_{j 2^{-m} \in W_{\xi}^{i}}$ is dominated by

$$
\sum_{j 2^{-m} \in W_{\xi}^{i}} C c_{\xi}^{2} j^{2(k-1)} 2^{-2 m k} a_{j}^{2}+C_{\alpha} c_{\xi}^{-2 \alpha} j^{-2 k \alpha} 2^{-2 m k \alpha} a_{j}^{-2 \alpha} .
$$

To finish, put $\beta=k-\frac{1}{4}, \alpha=3$, and let $a_{j}=c_{\xi}^{-1} j^{-\beta} 2^{m k}$ in (19) obtaining

$$
\sum_{j 2^{-m} \in W_{\xi}^{i}} \leq C \sum_{1}^{2^{m+1}} j^{2(k-\beta-1)}+j^{2 \alpha(\beta-k)} \leq C \sum_{1}^{\infty} j^{-3 / 2} .
$$

The terms $\sum_{j 2^{-m} \in V_{\xi}^{i}}$ can be handled similarly with $k=1$.

Since (20) is independent of $\xi$ and $m$, the proof is complete.

\section{REFERENCES}

[B] J. Barrionuevo, Estimates for some Kakeya type maximal operators, Trans. Amer. Math. Soc. 335 (1993), 667-682.

[C] A. Cordoba, Geometric Fourier analysis, Ann. Inst. Fourier (Grenoble) 32 (1982), 215-226.

[NSW] A. Nagel, E. M. Stein, and S. Wainger, Differentiation in lacunary directions, Proc. Nat. Acad. Sci. U.S.A. 75 (1978), 1060-1062.

[S] J. Stromberg, Maximal functions associated to uniformly distributed families of directions, Ann. of Math. (2) 108 (1978), 399-402.

[W] S. Wainger, Applications of Fourier transforms to averages over lower dimensional sets, Proc. Sympos. Pure Math., vol. 35, Amer. Math. Soc., Providence, RI, 1979.

Department of Mathematics and Statistics, University of South Alabama, Mobile, Alabama 36688

E-mail address: FJAB@USOUTHAL.BITNET 